Intersections

Canadian Journal of Music

Revue canadienne de musique
Intersections CANADAN TOURAA OP NUSIC

\title{
Michela Niccolai. 2011. La dramaturgie de Gustave Charpentier. Brepols, xxxiii, 540 p. ISBN : 978-2-503-54340-6
}

\section{Liouba Bouscant}

Volume 35, numéro 1, 2015

URI : https://id.erudit.org/iderudit/1038949ar

DOI : https://doi.org/10.7202/1038949ar

Aller au sommaire du numéro

\section{Éditeur(s)}

Canadian University Music Society / Société de musique des universités canadiennes

ISSN

1911-0146 (imprimé)

1918-512X (numérique)

Découvrir la revue

Citer ce compte rendu

Bouscant, L. (2015). Compte rendu de [Michela Niccolai. 2011. La dramaturgie de Gustave Charpentier. Brepols, xxxiii, 540 p. ISBN : 978-2-503-54340-6]

Intersections, 35(1), 159-164. https://doi.org/10.7202/1038949ar

Copyright ( C Canadian University Music Society / Société de musique des universités canadiennes, 2016
Ce document est protégé par la loi sur le droit d'auteur. L'utilisation des services d’Érudit (y compris la reproduction) est assujettie à sa politique d'utilisation que vous pouvez consulter en ligne.

https://apropos.erudit.org/fr/usagers/politique-dutilisation/ 
Plus qu'utile, ce volume était nécessaire. Il établit une assise solide pour les différentes histoires de la province du Québec où la musique intervient au cours de la première moitié du siècle dernier. On lui reconnaîtra la valeur de textes qui demeurent aisés à lire - sans pour autant sacrifier à la simplicité - et qui savent conserver l'attention. Mais son plus grand mérite - est-il besoin de le préciser - réside peut-être dans le fait qu'en positionnant le prix d'Europe au carrefour de tant de transformations ayant marqué le Québec moderne, il fait participer la musique aux transformations les plus concrètes d'une société et la dégage ainsi de son dommageable et trop récurrent statut de simple divertissement.

PAUl BAZIN

\section{RÉFÉRENCES}

Caron, Claudine . 2013. Léo-Pol Morin en concert. Montréal: Leméac. Villaggi, Jean-Pierre.2005. L’Administration publique québécoise et le processus décisionnel. Cowansville: Yvon Blais.

\section{BIOGRAPHIE}

Paul Bazin poursuit actuellement des études doctorales à l'Université McGill où il consacre ses recherches à la musique microtonale du compositeur Bruce Mather. Il est détenteur d'un baccalauréat en chant classique de l'Université de Sherbrooke (2010) ainsi que d'une maîtrise en musicologie de l'Université de Montréal (2013), dans le cadre de laquelle il s'est consacré à l'analyse des mélodies de Serge Garant. Ses travaux portent sur divers aspects de la vie musicale au Québec, incluant l'analyse et la critique.

Michela Niccolai. 2011. La dramaturgie de Gustave Charpentier. Brepols, xxxiii, 540 p. ISBN: 978-2-503-54340-6.

La recherche musicologique sur Gustave Charpentier (1860-1956) a longtemps été engourdie par l'indifférence du milieu musical à l'égard du défunt pourtant glorifié de son vivant. Elle connaît actuellement un fort regain d'activité: le cinquantième anniversaire de sa mort en 2006 a été célébré par le Musée de Montmartre, et Louise est entrée au répertoire de l'Opéra de Paris. À la thèse de Françoise Andrieux consacrée à Charpentier, l'éducateur du peuple, en 1985, puis aux travaux amorcés depuis les années 1990 par Jane Fulcher (1992), Steven Huebner (1995) et Barbara Kelly (2014), étudiant les opé-

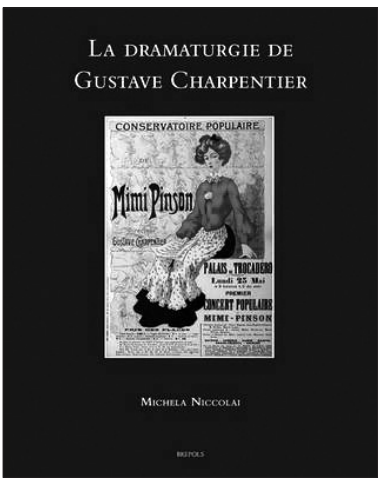
ras Louise et Julien sous l'angle social et politique dans les contextes de l'Affaire Dreyfus et de l'agitation anarchiste au tournant 
du XX $\mathrm{XX}^{\mathrm{e}}$ siècle, ainsi qu'à ceux de Jean-Christophe Branger (2007) sur le naturalisme dans l'opéra français du XIX ${ }^{\mathrm{e}}$ siècle, particulièrement dans Louise, se joint à présent une première monographie de synthèse sur ce musicien par Michela Niccolai. L'ouvrage se fonde sur des sources tendant à l'exhaustivité pour une majorité d'entre elles, inédites, notamment les Mémoires de ce compositeur, dont Niccolai prépare l'édition ${ }^{1}$, et de nombreuses notes et lettres autographes, iconographies et coupures de presse en lien direct avec la composition de ses œuvres. L'auteure présente en outre dans le premier chapitre l'inventaire qu'elle a effectué des «Fonds Charpentier», partagés entre la Bibliothèque Historique de la Ville de Paris, la Bibliothèque nationale de France, la bibliothèque personnelle de Charpentier conservée chez son petit-neveu Claude Charpentier et le Musée de Montmartre. Niccolai fournit la démonstration que les facteurs de cet état lacunaire de la connaissance au sujet de Charpentier résident dans l'ignorance de sa pensée sociale, formulée dans des écrits restés inédits, dans la difficulté d'accès aux partitions complètes et dans l'absence de prise en compte du contexte socio-historique de leur composition.

L'ouvrage développe en profondeur pour tous les chercheurs et étudiants qui s'intéressent à la période de la Troisième République, à l'opéra français, à l'engagement des musiciens, ainsi qu'à l'essor du disque et de la musique de cinéma, des thèmes à la fois indépendants, et ici reliés par un fil narratif monographique très cohérent: l'intégration des compositeurs au processus de républicanisme d'État, la constitution du répertoire d'art en plein air pour le peuple, l'évolution de l'esthétique de la mise en scène, les conséquences de l'apparition du disque, du cinéma et de la radio sur la création musicale, les relations entre musique «savante» et musique populaire.

L'hypothèse que veut vérifier l'auteure est celle d'un musicien intégralement dramaturge social. Tels sont l'originalité et l'atout de ce volume : partir de l'objet musical opératique auquel est inhérente la notion de dramaturgie et en induire la conscience sociale et l'engagement de Charpentier. La qualité de dramaturge inhérente à l'identité musicienne de Charpentier trouve une résonance jusqu'à son vécu réel et ses réalisations extramusicales, tel que le déclare Niccolai: «La dramaturgie du compositeur s'est manifestée à nous à travers plusieurs aspects : la réalisation d'un spectacle populaire, Le Couronnement de la muse, d'un opéra social, Louise, la fondation d'une initiative active, le Conservatoire de Mimi Pinson, enfin, la passion pour la modernité. Les nouveaux médias» (Niccolai, p. xxxi). L’unité musicale qui s'observe entre toutes ces réalisations est le fruit de la volonté d'unification sociale et de cohésion nationale sous le signe de l'émancipation féminine et de l'éducation dans les couches ouvrières, de la part d'un enfant du peuple qui a vu son ascension sociale permise par les institutions de la Troisième République.

Ceux qui sont sensibles à la question de l'influence de Wagner dans l'opéra de Charpentier pourraient être déçus: Niccolai annonce d'entrée de jeu les limites de son étude, consciemment choisies, dans son introduction: elle ne s'attachera pas au wagnérisme de Charpentier. Pourtant, elle nous apprend au

1 À paraître. 
cours de son travail que ce sont en grande partie les fêtes wagnériennes, outre Jules Michelet, qui marquent en profondeur toute la démarche compositionnelle sociale de Charpentier, et ce, dès son séjour à la Villa Médicis à Rome.

D'emblée, l'auteure pose l'axiome selon lequel chez Charpentier, l'œuvre dramaturgique est avant tout le reflet de la vie: ce musicien perçoit et s'intègre à son environnement en composant. Originaire du nord-est de la France, Charpentier devient un Montmartrois et d'adoption et dans l'âme, amoureux du mode de vie de l'artiste bohème arpentant la célèbre butte parisienne. Il met en scène musicalement la symbolique sociale et politique de celle-ci en une «image presque photographique d'un monde qui est au sommet de la célébrité culturelle, et qui disparaîtra avant la Première Guerre mondiale [...] peinte [...] dans toute sa substance et son essence» (p. 83).

Michela Niccolai se propose d'élucider l'énigme suivante: comment et pourquoi un succès de masse à message social et une esthétique jugée par ses contemporains comme une alternative au wagnérisme et au symbolisme sont-ils ensuite perçus pendant des décennies comme un phénomène de mode contingent n'ayant pas fait école, au contenu pittoresque, localiste et suranné?

Cet ouvrage développe la thèse selon laquelle Charpentier est le seul musicien de son époque à exprimer les «enjeux de la modernité» sociale enclenchée par la société industrialisée à la fin du XIX ${ }^{\mathrm{e}}$ siècle. Artiste engagé, il apparaît comme un acteur de l'histoire, traduisant les revendications de la "société finde-siècle »: l'union libre, l'égalité des classes, l'émancipation des femmes. Sans adhérer à un parti politique, le musicien montmartrois est animé d'un idéal socialiste utopique, proche du politicien Joseph Paul-Boncour. Au contact de celui-ci, il aurait été influencé - Michela Niccolai évoque seulement cette hypothèse dans son introduction - par la franc-maçonnerie.

Charpentier, par ailleurs, s'affirme comme un cas singulier de compositeur acteur de la cohésion nationale. Représentant officiel du régime et de l'art républicains en tant que membre de l'Institut de France, appartenant par conséquent à la nomenklatura, il est cependant marginal par son appartenance de classe et de cœur à la classe prolétarienne combattant pour l'équité des droits sociaux.

L'ouvrage est construit en quatre parties, chacune correspondant à des aspects particuliers de la problématique:

I. Histoire de 10 ans d'activité créatrice (1890-1900)

II. Un demi-siècle de travail consacré à l'Éducation du peuple à l'Art et à la Beauté

III. Création et analyse d'un mythe: de Louise à Mimi

IV. De l'Opéra-comique à l'industrie du spectacle

Dans l'introduction, la musicologue explique que les origines ouvrières du Nord de la France du compositeur, sa pratique musicale, adolescent, dans les orphéons, ainsi que son expérience de travail auprès d'un employeur de filature au paternalisme social soucieux de faire pratiquer la musique à ses ouvriers, sont associées à une sympathie profonde pour le socialisme utopique. Dès lors, le musicien professionnel entrant au Conservatoire dans la classe de violon en 1879, puis dans la classe de composition de Massenet en 1885, Prix de Rome 
en 1887 , se donne corps et âme à la défense des droits des musiciens au sein du mouvement syndicaliste puis à l'éducation musicale des jeunes ouvrières.

La première partie dépeint le contexte sociopolitique, artistique et culturel fin-de-siècle relié à la genèse des compositions de Charpentier, pour la plupart méconnues: Louise, Le Couronnement de la Muse, essentielle et oubliée, et une partition ignorée, la symphonie Munich, composée entre 1910 et 1912. Lauteure place en avant-plan les deux cultures artistiques liées au peuple dont Charpentier se nourrit: la littérature et l'art savants tournés vers le peuple et une culture d'essence populaire, sur fonds de socialisme et de syndicalisme ouvrier. De surcroît, les années de gestation des œuvres de Charpentier sont marquées par les relents de l'anarchisme et des attentats de Ravachol, qui fascinent un milieu bohème rebellé contre les injustices sociales et la répression politique réactionnaire. Cependant, c'est le syndicalisme enfanté par l'anarchisme avorté qui séduit Charpentier. Michela Niccolai relate la participation de Charpentier dans la création et l'action de la Chambre syndicale musicale vers 1900 et la grève générale.

Michela Niccolai montre comment, de Louise, naît en 1897 Le Couronnement de la muse, symbole républicain de la réconciliation de l'art et du peuple, conçue pour être jouée par les ensembles amateurs dans tout le pays afin de répandre les valeurs sociales et culturelles du régime républicain mais aussi pour que la Beauté soit réalisée par le peuple lui-même, et non qu'elle lui soit seulement présentée.

La deuxième partie est minutieusement dédiée à la genèse de l'écriture du livret et de la musique de l'opéra Louise et à ses mises en scène, notamment celle d'Albert Carré, leurs contextes esthétiques et les influences venues du théâtre fin-de-siècle. Louise, bien que restée sans descendance n'est en aucune façon une carte postale musicale pittoresque. Michela Niccolai montre que Charpentier souhaite réaliser un opéra parisien lyrique, dans lequel les héros sont humbles et soumis aux «exigences de la vie sociale» (p. 152), et dont le livret est tributaire de l'influence des thèses et des romans naturaliste de Zola, notamment Une page d'amour personnifiant Paris.

La troisième partie induit de l'œuvre artistique l'engagement social de l'artiste. Les diverses activités du Conservatoire populaire d'enseignement de la musique vocale, instrumentale et de la danse classique, dans une optique plus générale de création et de pratique de l'art "par et pour le peuple» (Charpentier cité p. 332), la formation du mythe de Mimi Pinson et de la Muse, son rayonnement humanitaire durant la Première Guerre mondiale et sa portée politique, puis esthétique et commerciale, l'utilisation socialiste républicaine à l'occasion du Couronnement de la Muse sont l'objet de ce chapitre. Celui-ci se clôt par une analyse très convaincante des causes esthétiques et contextuelles de l'échec de Julien, créé en 1913, malgré les recettes très satisfaisantes.

La quatrième partie, "De l'Opéra-comique à l'industrie du spectacle", analyse la façon dont Charpentier, épris de progrès technique et amateur de cinéma, sans pour autant vouloir céder la paternité de son œuvre, entreprend d'adapter ses œuvres aux nouveaux médias, le disque, la radio et le cinéma. Charpentier est par conséquent l'un des pionniers du concept des œuvres «ouvertes» aux 
multiples formats possibles. Niccolai étudie notamment le cas de Louise, premier opéra enregistré au disque 78 tours, en 1935, recevant le Grand Prix du Disque en 1936, et la façon dont Charpentier en modifie le contenu et la substance pour en obtenir une version radiophonique.

Michela Niccolai relève avec brio le défi de la complexité d'un va et vient esthétique et social entre les œuvres et le réel, et leurs interactions. Il apparaît, à l'issue de la lecture de son livre, que Louise est l'œuvre matrice et la plus représentative de la poétique et du message social de Charpentier. Par ailleurs, Niccolai démonte toutes les qualifications, à l'égard de Louise, de populisme et d'opéra d'un «milieu»-la classe ouvrière, en prouvant que la volonté et l'action de Charpentier en son temps sont bien la cohésion sociale républicaine. Ressort de cette monographie un fait frappant: tout est prémédité et réfléchi par Charpentier dès son séjour à la Villa Médicis en 1888.

Admirable est la plongée dans les contextes politiques, sociaux et esthétiques, et leurs imbrications avec les enjeux technologiques naissants. À l'instar de Zola dans ses romans naturalistes, l'auteure évince habilement tout en le suggérant, le métadiscours théorique mettant en relation de cause à effet le milieu social et l'hérédité sur la formation de l'identité intellectuelle, politique et artistique. Elle utilise l'axiome psychologique et esthétique: Charpentier vit la musique qu'il compose. Sa démarche d'artiste met en musique sa vie et ses idéaux, la rue et la foule - attitude somme toute naturaliste ancrée solidement dans son époque.

Cependant, des choix sont inévitables: en plaçant Charpentier, musicien social de la Troisième République et sa muse Louise, cristallisations humaine et artistique d'un contexte social et musical du tournant du $\mathrm{XX}^{\mathrm{e}}$ siècle français, au cœur de son portrait en vitrail, Niccolai privilégie l'éclairage sur certaines problématiques selon les époques abordées et laisse le rapport précis entre Charpentier, musique pour le peuple et politique de gauche dans les années trente dans la pénombre, ne mentionnant l'activité politique de Charpentier dans les années trente qu'en conclusion. Enfin, aurait été utile une mise en perspective légèrement plus développée avec le parti politique socialiste, l'idéologie socialiste utopique et le paternalisme industriel au début de la III République ainsi quavec la source saint-simonienne chez les musiciens sociaux du $\mathrm{XIX}^{\mathrm{e}}$ siècle, puis avec le communisme en France dans les années trente.

Liouba Bouscant

\section{RÉFÉRENCES}

Andrieux, Françoise. 1985. "Gustave Charpentier, artiste social: Contribution à l'étude de l'éducation musicale populaire». Thèse de doctorat, Université Paris IV «Sorbonne».

Branger, Jean-Christophe. 2007. «Du naturalisme dans Louise», dans Louise, programme de salle. Paris: Opéra-Bastille, p. 68-73.

Fulcher, Jane. 1992. "Charpentier's operatic Roman musical as Read in the Wake of Dreyfus Affaire», 19th Century Music, vol. XVI, no 2, p. 161-180. 
Huebner, Steven. 1995. «Between Anarchy and the Box Office: Gustave Charpentier's Louise", 19th Century Music, vol. XIX, n 2, p. 136-160.

Kelly, Barbara. 2014. «Vie parasite du poète: Art et recyclage dans Julien de Charpentier ", dans Le naturalisme sur la scène lyrique, Branger et Ramaut, dir. Saint-Étienne: Presses universitaires de Saint-Étienne, p. 271-283.

\section{BIOGRAPHIE}

Liouba Bouscant est agrégée de musique, diplômée du Conservatoire National Supérieur de Musique et de Danse de Paris en Analyse, Histoire de la musique et Esthétique, et docteur en musicologie. Ses travaux portent sur les rapports entre musique et politique au $\mathrm{XX}^{\mathrm{e}}$ siècle ainsi que sur l'esthétique, la rhétorique et l'herméneutique musicales. Elle a publié une étude des quatuors à cordes de Chostakovitch (2004, éditions L'Harmattan) et prépare un ouvrage sur l'engagement politique et social du compositeur Charles Koechlin (1867-1950).

Bartoli, Jean-Pierre et Jeanne Roudet. 2013. «L'Essor du romantisme: la fantaisie pour clavier: de Carl Philipp Emanuel Bach à Franz Liszt ». MusicologieS. Paris: Vrin, 387 p. ISBN 978-2-7116-2473-7

L'histoire de la musique fait traditionnellement apparaître la production musicale entre 1750 et 1850 comme inexplicablement en retard sur les autres arts. Le défi que Jean-Pierre Bartoli et Jeanne Roudet relèvent avec brio dans ce livre est de renverser cette conception en montrant que la fantaisie pour clavier est un vecteur de continuité esthétique à cette période. Au passage, ils débroussaillent un pan majeur de la littérature pour clavier, souvent injustement négligé.

Les auteurs empruntent au linguiste Nicolas Ruwet une disposition dite paradigmatique, qui est essentiellement une présentation schématique de la forme et qui en explicite les différents retours thé-

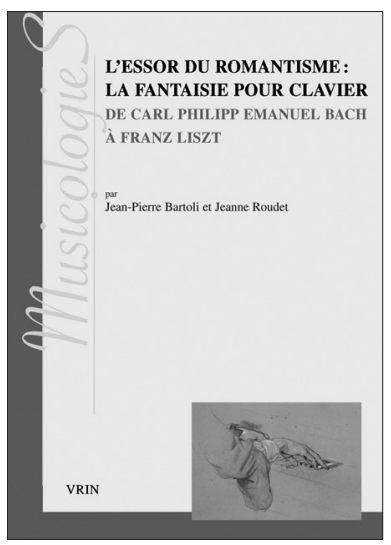
matiques, motiviques ou autres. Les analyses musicales du présent ouvrage étant essentiellement formelles, le choix de la méthode aide grandement à la compréhension en permettant de saisir d'un coup d'œil certains arguments avancés par les auteurs. Nous déplorons cependant la révision lacunaire de ces schémas, qui portent parfois à confusion ${ }^{1}$. Néanmoins, rehaussant considérablement la

1 Par exemple, «réexposition » est abrégé de quatre manières différentes dans un même schéma (p. 150); l'italique et le romain se mélangent sans raison apparente (p. 312); lettres grecques et romaines se succèdent sans explication pour identifier les motifs (p. 185, 191, 283); les lettres « $\mathrm{S}$ » et « $\mathrm{D}$ » remplacent arbitrairement les fonctions IV et $\mathrm{V}$ dans le plan tonal (ce qui donne S/S et D/D pour IV/IV et V/V), p. 157. 